\title{
Variable density vortex ring dynamics in sharply stratified ambient fluids
}

\author{
Roberto Camassa, ${ }^{1,2}$ Daniel M. Harris, ${ }^{1,2}$ David Holz, ${ }^{3}$ Richard M. McLaughlin,,${ }^{1,2, *}$ \\ Keith Mertens, ${ }^{3}$ Pierre-Yves Passaggia, ${ }^{2,4}$ and Claudio Viotti ${ }^{5}$ \\ ${ }^{1}$ Department of Mathematics, University of North Carolina at Chapel Hill, \\ Chapel Hill, North Carolina 27599, USA \\ ${ }^{2}$ UNC Joint Fluids Lab, University of North Carolina at Chapel Hill, Chapel Hill, North Carolina 27599, USA \\ ${ }^{3}$ Leap Motion, 333 Bryant Street, San Francisco, California 94107, USA \\ ${ }^{4}$ Department of Marine Sciences, University of North Carolina at Chapel Hill, \\ Chapel Hill, North Carolina 27599, USA \\ ${ }^{5}$ Miravex, 11 St. Stephen's Green, Dublin 2, Dublin, Ireland \\ (Received 5 August 2016; published 12 September 2016)
}

\begin{abstract}
This paper is associated with a poster winner of a 2015 APS/DFD Milton van Dyke Award for work presented at the DFD Gallery of Fluid Motion. The original poster is available from the Gallery of Fluid Motion, http://dx.doi.org/10.1103/APS.DFD.2015.GFM.P0050
\end{abstract}

DOI: 10.1103/PhysRevFluids.1.050503

Vertical density stratification is a key element in the dynamics of geophysical fluids such as lakes, oceans, and the atmosphere. Often, the vertical transport of matter through such density profiles results in self-propagating coherent vortex structures and their breakdown or persistence can greatly affect mixing of the surrounding fluid. Such processes can contribute to the formation of trapped plumes in the ocean and atmosphere, as occurred for instance in the 2010 Deepwater Horizon oil spill in the Gulf of Mexico [1]. To gain some understanding of the vortex stability it is advantageous to consider the simplest possible setup involving a single vortex. The case of a density matched vortex traveling in a homogeneous fluid is the scenario most commonly examined in the literature. Here we consider the more complex variable density setup involving a vortex settling in a stratified ambient fluid, with an initial vortex density greater than the bottom fluid. In our experiments, this stratification is characterized by three regions in a $10 \mathrm{~cm} \times 10 \mathrm{~cm} \times 19 \mathrm{~cm}$ acrylic tank: a constant density fresh water top layer $(0.9998 \mathrm{~g} / \mathrm{cc})$, approximately $2 \mathrm{~cm}$ thick, a linear stratification of $5 \mathrm{~cm}$ thickness, and a constant deep layer of salty water $(1.025 \mathrm{~g} / \mathrm{cc})$. The diameter of the vortex is initially approximately $1 \mathrm{~cm}$ and generally grows during the course of the experiment (as noticed in the computational and experimental study [2]). In Fig. 1, we show a sequence of snapshots (side view: left and center columns; bottom view: right column) documenting the vortex ring evolution and its breakup as it passes into and through the stratified region. A Nikon D4 camera equipped with a $105 \mathrm{~mm}$ microNikkor lens, focused below the stratified region, was used to capture images. The color shadowgraphs are visualized using a parallel light source illuminating the vortex ring from the top and the side. The heavy vortex ring is created by a rhodamine dyed salt water drop $(1.05 \mathrm{~g} / \mathrm{cc})$, generated by a Harvard syringe infusion pump model 975 connected to a stainless steel needle of $2 \mathrm{~mm}$ external diameter located $25 \mathrm{~mm}$ above the surface, following generation methods documented in the literature [2,3]. The presented pictures are finally obtained by inverting the color map, resulting in a blue background and a green vortex ring. As the vortex ring travels into the stratified region, it entrains light, top-layer fluid into dense bottom fluid. This enhances the vortex ring deceleration, leading to the observed vortex breakup which can be attributed mostly to a Rayleigh-Taylor type instability. The dense core

\footnotetext{
*rmm@email.unc.edu
}

Published by the American Physical Society under the terms of the Creative Commons Attribution 3.0 License. Further distribution of this work must maintain attribution to the author(s) and the published article's title, journal citation, and DOI. 

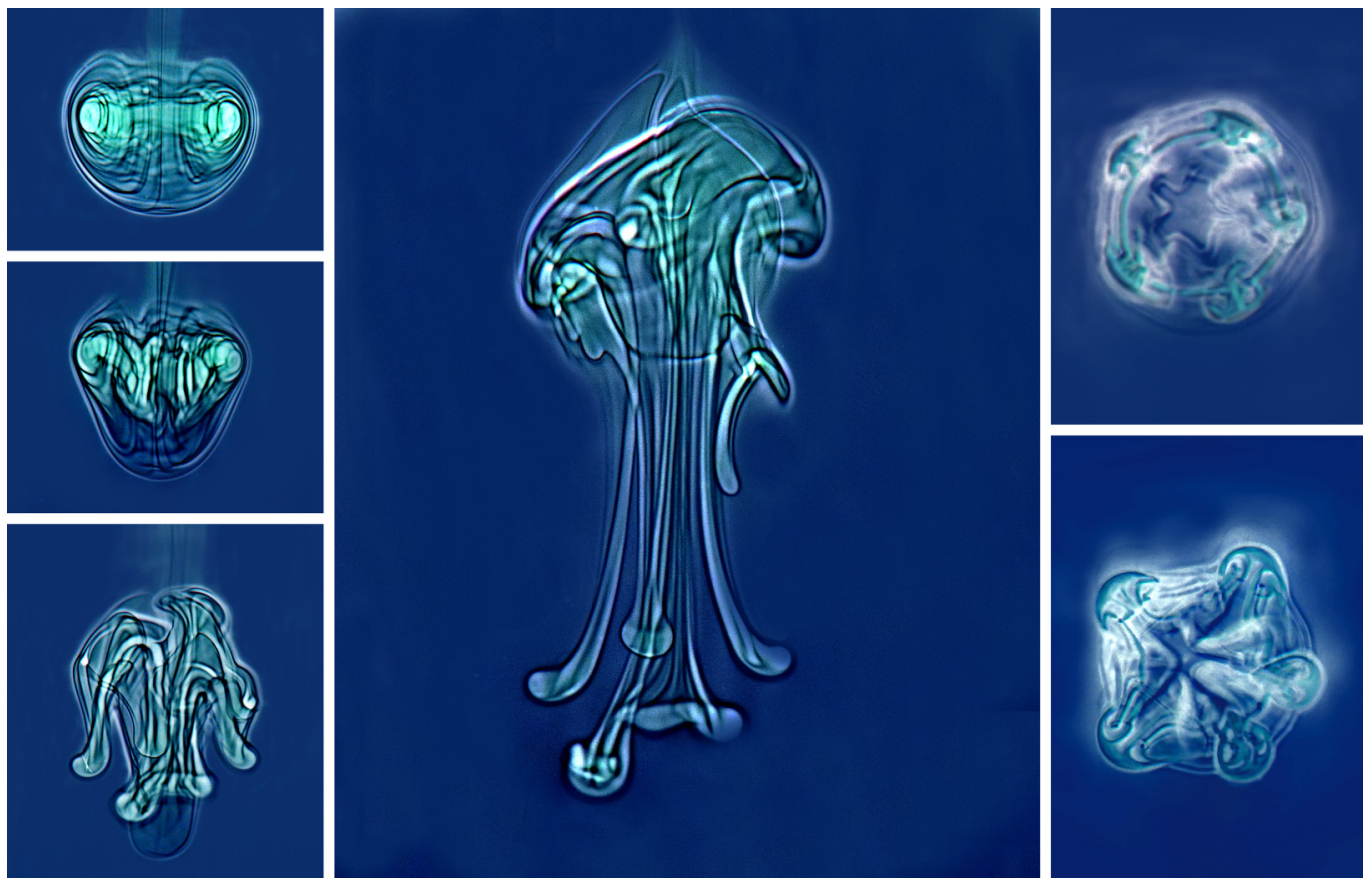

FIG. 1. Left column: Side view of the vortex entering the bottom denser layer and the progression of vortex breakup. Center panel shows the later time structure, with the entrained top-layer fluid returning upward, though the center of the ring, creating a "jellyfish" pattern. Right column: Bottom view showing the pentagonal symmetry of the growth of the underlying instability. See the original poster at http://dx.doi.org/10.1103/APS.DFD.2015.GFM.P0050

fluid settles, while a mixture of mostly top-layer fluid generates a rising plume forming the "jellyfish"like cap. The "tentacles" of heavy core fluid following the vortex breakup bear resemblance to those recently reported [4] for the case of vortex rings with a core density larger than homogeneous ambient fluid, while the rising plume in the form of a cap is due to the ambient fluid stratification.


FIG. 2. Comparison of four- and fivefold symmetry of the vortex breakup visualized from below. 


\section{VARIABLE DENSITY VORTEX RING DYNAMICS IN . . .}

The Rayleigh-Taylor instability mechanism provides a simple estimate for predicting the $N$-fold symmetry leading to vortex breakup by selecting a most unstable viscous wavelength. This suggests the possibility of obtaining different than pentagonal shapes. An example contrasting the pentagonal symmetry with a diamond configuration is presented in Fig. 2 (black and white) where the height of the injector was increased by $5 \mathrm{~mm}$. This results in a faster and smaller initial vortex ring where only four folds are observed in our stratified system.

The authors would like to acknowledge J. R. Bowlin for assistance with the artwork. The research was supported by NSF RTG DMS-0943851, NSF CMG ARC-1025523, NSF DMS-1009750, NSFOCE-1155558, NSF DMS-1517879, and the office of Naval Research for support through ONR DURIP N00014-12-1-0749.

[1] R. Camassa, Z. Lin, R. M. McLaughlin, K. Mertens, C. Tzou, J. Walsh, and B. White, Optimal mixing of buoyant jets and plumes in stratified fluids: Theory and experiments, J. Fluid Mech. 790, 71 (2016).

[2] R. Camassa, S. Khatri, R. McLaughlin, K. Mertens, D. Nenon, C. Smith, and C. Viotti, Numerical simulations and experimental measurements of dense-core vortex rings in a sharply stratified environment, Comput. Sci. Discovery 6, 014001 (2013).

[3] D. S. Chapman and P. R. Critchlow, Formation of vortex rings from falling drops, J. Fluid Mech. 29, 177 (1967).

[4] M. Shimokawa, R. Mayumi, T. Nakamura, T. Takami, and H. Sakaguchi, Breakup and deformation of a droplet falling in a miscible solution, Phys. Rev. E 93, 062214 (2016). 